\title{
Flux de sève et alimentation hydrique de cerisiers irrigués ou non en localisation
}

\author{
B Cabibel $^{1^{*}}, \mathrm{C}$ Isbérie $^{2}$ \\ avec la participation technique de J Horoyan*
}

'Unité de science du sol, Inra, domaine Saint-Paul, site Agroparc, F-84914 Avignon cedex 9;
${ }^{2}$ Équipe irrigation, division HIAX, Cemagref. BP 3I, F-I36I2 Aix-en-Provence cedex I, France

(Reçu le 29 décembre 1995 ; accepté le 7 février 1997)

\begin{abstract}
Summary - Sap flow and water consumptive use in cherry trees irrigated or not irrigated under trickle irrigation. Hydric behaviour of cherry trees, irrigated under trickle irrigation and non-irrigated (table I), are investigated. Irrigation rate is controlled by tensiometry. Sap fluxes in cherry trees are measured by a thermal method, hourly branch diameter variations by micromorphometry, and water soil transfer by neutronometry and tensiometry (fig 1). It has been shown that: i) a general heterogeneity of sap fluxes is present in different sectors of a cherry tree trunk section (fig 2; table II). The heterogeneity is closely correlated and induced by the spatial and temporal heterogeneity of different soil sector wetting in relation to local root bundles (fig 3); under irrigation, the ratio between extreme fluxes, measured in a trunk section, is close to the ratio between the two sources of water in the soil, irrigation and the natural soil water contribution; ii) sap flow in trunks, whatever the hydric conditions may be, is essentially a function of instantaneous availability of soil water, the effect of ETP being virtually negligible; iii) a heterogeneity of diameter microvariations is present in branches of non-irrigated trees and not in irrigated trees (figs 4 and 5). It has been concluded that preferential flow pathways exist in the soil-plant system.

The relative participation, in time, of internal water tree reserves and sap flows to evapotranspiration (figs 6 and 7), and the existence of water stress in trees or the satisfaction of their real water requirements as a function of different water supplies have also been shown. It is concluded that methodology used is not only a good tool for characterising water relations and water stress of trees but also for upgrading trickle irrigation scheduling of cherry tree orchards, especially if these measures are continuous and self-acting.
\end{abstract}

sap flow / diurnal stem shrinkage / cherry tree / trickle irrigation / water stress / irrigation scheduling

Résumé - Le comportement hydrique de cerisiers irrigués ou non en localisation est étudié en continu. Les apports d'eau à la culture sont pilotés par tensiométrie. Les flux de sève brute dans le tronc des arbres sont quantifiés par la méthode thermique du flux radial, les variations de diamètre des branches sont mesurées par des capteurs de microdéplacement (dendrométrie), et les transferts d'eau dans le sol sont estimés par humidimétrie neutronique et tensiométrie. On montre : i) qu'il existe une hétérogénéité générale des flux dans la section des troncs. Cette hétérogénéité est trouvée liée à l'hétérogénéité spatiale et temporelle des potentialités locales d'alimentation hydrique du sol vis-à-vis des racines présentes localement : sous irrigation la valeur du rapport entre les flux extrêmes mesurés traversant la sèction du tronc est proche de celle du rapport entre les deux sources d'alimentation de l'arbre, l'irrigation et la contribution naturelle du sol ; ii) que les flux dans les troncs, quel que soit le traitement hydrique appliqué à l'arbre, sont essentiellement fonction de la disponibilité instantanée de l'eau du sol ; l'irrigation étant toujours très inférieure à la demande climatique, l'influence de l'ETP n'apparaît que peu, car la transpiration s'est adaptée aux quantités d'eau apportée ; iii) qu'il existe un comportement homogène des capteurs de déplacement situés dans l'arbre irrigué, alors qu'une hétéro-

* Correspondance et tirés à part

Tél : (33) 0490316000 ; fax : (33) 0490316244 
généité se manifeste dans l'arbre non irrigué ; iv) qu'il existe des voies préférentielles de flux dans le système sol-racine-plante. On met de plus en évidence la participation relative des réserves en eau de l'arbre et des flux racinaires à l'évapotranspiration et son évolution dans le temps, en fonction des deux traitements hydriques appliqués aux arbres. On conclut que l'approche utilisée s'avère être un bon outil de caractérisation de l'état hydrique des arbres et de diagnostic du stress hydrique, mais offre également des éléments d'analyse permettant d'améliorer les modalités de conduite de l'irrigation localisée en arboriculture fruitière, et cela d'autant mieux que les mesures sont continues et automatisables.

flux de sève / variation de diamètre du tronc / cerisier / irrigation localisée / stress hydrique / pilotage de l'irrigation

\section{INTRODUCTION}

Le cerisier, pour fleurir et produire, a des besoins en froid relativement importants. Il est donc le plus souvent présent dans des régions septentrionales peu ou pas soumises à l'irrigation. Dans les régions où il est susceptible d'être irrigué, régions à climat relativement doux au printemps, la récolte s'effectue tôt en saison, en mai ou juin. À cette période, l'arbre peut presque toujours compter sur les ressources en eaux naturelles pour son alimentation hydrique, ce qui implique que la culture du cerisier n'est en général pas une culture irriguée. Lorsqu'elle est pratiquée, l'irrigation a pour objectif essentiel la survie du verger en cas de fortes sécheresses estivales.

Il n'existe que peu d'études concernant l'irrigation de cette espèce fruitière, notamment sous irrigation localisée. Les études faites montrent une fréquente indépendance des résultats de la récolte vis-à-vis de l'irrigation de l'année, donc l'influence faible ou nulle, à court terme, des apports d'eau sur le rendement. Certaines soulignent néanmoins que l'irrigation localisée est la technique d'apport d'eau qui induit la meilleure efficience agronomique de l'eau d'irrigation et qu'elle a une incidence favorable sur la physiologie et le développement de l'arbre. En sa présence il y a une augmentation en éléments minéraux dans les feuilles, un grossissement des troncs et un allongement des pousses (Sizov et Lastreb, 1976 ; Smith et Kenworthy, 1979 ; Kesner et al, 1985). Elle favorise également le rendement cumulé d'associations (greffon + porte-greffe) vigoureuses et peu productives, notamment lorsque l'irrigation est trop réduite ou volontairement rationnée (Soing et Mandrin, 1992). Il apparaît donc qu'un apport d'eau qui minimiserait les risques de stress hydrique, effectué avant les périodes de sécheresse, devrait avoir à long terme, notamment sous climat méditerranéen, une action bénéfique sur la récolte.
Dans le cadre des projets hydrauliques régionaux en cours de réalisation ou d'achèvement en Provence (sud de la France), 1000 ha de cerisiers se trouvent en cours d'équipement d'irrigation. Les sécheresses, fréquentes ces 15 dernières années, y ont en effet entraîné des dégâts importants aux vergers pouvant aller jusqu'à leur quasi-mortalité. Ces dommages sont incompatibles avec l'intensification actuellement voulue de cette culture de sorte que l'irrigation apparaît non seulement comme un facteur essentiel de survie des arbres mais aussi comme un facteur nécessaire de régularisation et d'accroissement des rendements.

Dans le cadre des études en cours pour déterminer les besoins réels en eau du cerisier sous irrigation localisée (Isbérie, 1992), il devenait nécessaire d'initier des recherches spécifiques sur le comportement hydrique général du cerisier en situation irriguée et non irriguée. Dans cette étude, les objectifs visés sont, en premier lieu, de caractériser et d'estimer, notamment en conditions d'humectation hétérogène du sol : i) l'hétérogénéité spatiale et temporelle du prélèvement de l'eau du sol par les racines des arbres et des flux de sève brute dans la section des troncs ; ii) de confirmer l'existence de voies préférentielles pour les flux hydriques reliant un secteur du sol, le faisceau racinaire qui y est contenu et le secteur du tronc en relation avec ce faisceau racinaire. Il s'agit de vérifier si cette hypothèse mise en évidence in situ sur une espèce fruitière à pépins sous irrigation localisée (Do, 1987 ; Cabibel, 1994) est généralisable à l'espèce à noyau étudiée. En second lieu, il s'agit d'estimer la participation relative des réserves internes en eau des parties ligneuses des arbres à l'évapotranspiration, le rythme de cette participation et le degré de régénération des réserves utilisées. La finalité est ici de juger du degré de satisfaction des besoins en eau des arbres et de l'efficacité des apports d'eau effectués, notamment lorsque ceux-ci sont soit inférieurs à la demande climatique soit rationnés. 
Cet ensemble de données devraient permettre de mieux préciser la réaction de l'arbre à des stress hydriques pouvant exister. Il permettra de plus de mieux raisonner l'irrigation quant à ses paramètres techniques (positionnement et nombre de goutteurs par arbre) et ses paramètres hydriques (dose, fréquence et heure de déclenchement des apports d'eau) dans les vergers à grand écartement.

Pour parvenir à ces objectifs, une démarche expérimentale originale est mise en œuvre. Elle s'appuie simultanément sur la méthodologie classique de la science du sol pour la mesure des transferts d'eau dans le sol et sur l'utilisation en continu de capteurs physiologiques pour caractériser et estimer les flux hydriques dans l'arbre.

\section{MATÉRIEL ET MÉTHODES}

\section{Le verger expérimental}

Planté en 1977, le verger est constitué de cerisiers Bigarreaux Napoléon greffés sur Sainte-Lucie SL 64. Les arbres, distants de $6 \mathrm{~m}$ sur la ligne de plantation et de $7 \mathrm{~m}$ entre rangs, sont conduits en grands gobelets plein vent. Leur frondaison couvre $90 \%$ du sol à 14 heures (heure locale soit à midi solaire). Le verger est installé sur un sol de coteau, colluvions de sables fins limoneux d'épaisseur variable sur substratum sableux. Le sol est désherbé, par passage de rotavator entre rangs et chimiquement sur le rang. Les deux arbres étudiés d'une hauteur de $4,5 \mathrm{~m}$ ont des troncs de $0,70 \mathrm{~cm}$ de haut. L'arbre irrigué est situé en bordure nord du verger, séparé d'une vigne adulte par une bande de sol de $6 \mathrm{~m}$ non enherbée. L'arbre non irrigué, en bordure est du verger, est bordé par un chemin caillouteux. Le système d'irrigation localisé, mis en place à la plantation, comporte, par arbre, deux goutteurs de $4 \mathrm{~L} \cdot \mathrm{h}^{-1}$, situés sur la ligne de plantation à $1 \mathrm{~m}$ de part et d'autre du tronc (fig $1 \mathrm{~A}$ ). L'irrigation vise à maintenir, par arbre et tout au long de la période d'apport d'eau, deux volumes de sol humide les plus réguliers possibles. Elle débute à 16 heures (heure locale). Elle est pilotée journalièrement par tensiométrie (Peyremorte, 1980). Le principe de pilotage retenu est de maintenir, grâce aux apports d'eau, une valeur la plus régulière possible pour le potentiel matriciel de l'eau du sol, potentiel mesuré quotidiennement le matin, à l'aide de tensiomètres placés en périphérie des zones de sol humide à créer ; cinq arbres irrigués sont équipés d'un tensiomètre de pilotage, implanté à $50 \mathrm{~cm}$ de distance latérale du goutteur et à $30 \mathrm{~cm}$ de profondeur (fig 1B). Les doses d'eau sont raisonnées en fonction de la valeur médiane des potentiels trouvés, de façon à ce que la tension observée sur les tensiomètres de contrôle reste dans une gamme de mesure comprise entre 30 et $60 \mathrm{kPa}$, potentiels correspondant, en dessèchement, à des teneurs en eau volumique de 21,5 et $18 \%$. Les volumes de sol humide ainsi créés représentent de 0,3 à $0,8 \mathrm{du}$ volume total de sol étudié contribuant à l'alimentation hydrique de l'arbre, volume total de sol assimilé à celui qui, ayant en surface $42 \mathrm{~m}^{2}$, est exploré par l'ensemble du système racinaire de l'arbre, sur la profondeur de mesure de la teneur en eau $(280 \mathrm{~cm})$. Les doses d'irrigation (I) contrôlées journalièrement par compteur volumétrique et les quantités d'eau apportées par goutteur (lg) sont données au tableau I.

\section{Le climat}

De type méditerranéen, le climat de l'année 1989 a été caractérisé par une sécheresse exceptionnelle. Une pluviométrie hivernale faible et des ETP de printemps élevées ont conduit fin mai à un stock d'eau utilisable par la culture de $60 \mathrm{~mm}$, stock estimé sur une profondeur de sol de $2,80 \mathrm{~m}$. La médiane correspondante, calculée sur une période de 8 ans, étant de $210 \mathrm{~mm}$, le déficit en eau est donc en 1989 très important. Les paramètres climatiques sont obtenus, à proximité du verger, à la station météorologique du réseau Cirame de Cabrières d'Avignon. L'ETP est calculée par la formule de Penman par les services du Cirame. L'ETP journalière et le rayonnement global sont donnés au tableau 1 .

\section{Quantification des transferts d'eau dans le sol}

Les teneurs en eau volumique en fonction de la profondeur $\left(z_{\mathrm{n}}\right)$ et du temps $(t), \theta\left(z_{\mathrm{n}}, t\right)$, nécessaires au calcul de l'évolution des stocks d'eau disponibles pour la culture, sont mesurées à l'aide. d'un humidimètre densimètre neutron/gamma de profondeur SOLO 40 (Nardeux Humisol, France). Deux sites de mesures sont suivis : un site irrigué (l) et un site non irrigué (NI). Chaque site comprend quatre tubes de mesures (aluminium AG3, diamètre 41-45). Ils sont disposés sur la ligne de plantation, à respectivement 130, 180, 230 et 280 $\mathrm{cm}$ de l'arbre (fig 1A et C). Le choix des distances d'implantation résulte du fait que la présence des charpentières rendait impossible l'implantation de tube plus proche du tronc. Cependant des mesures, 


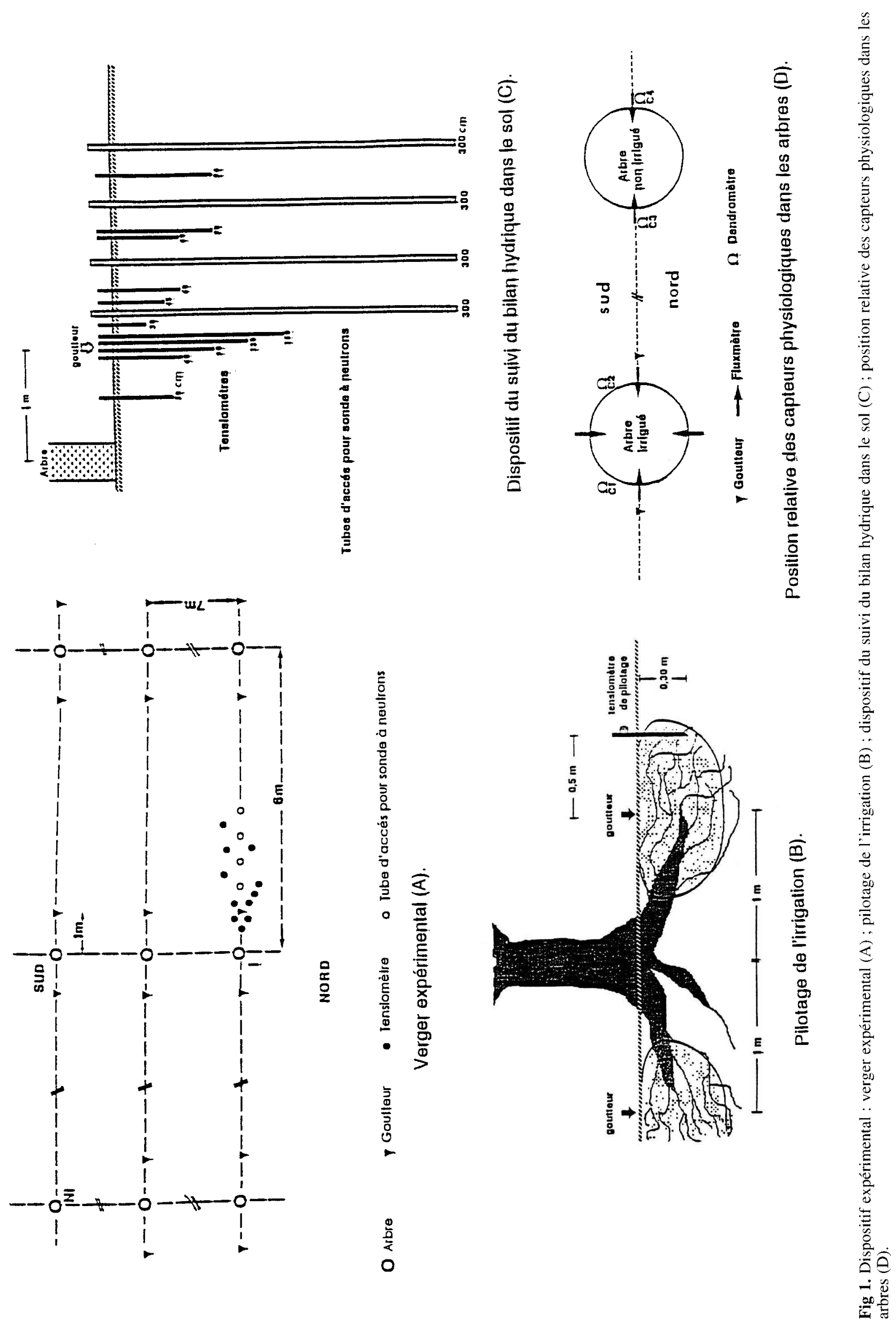


Tableau I. Évapotranspiration potentielle (ETP) et rayonnement global (Rg), alimentation hydrique des arbres.

\begin{tabular}{lccccccc}
\hline Date & $\begin{array}{c}E T P \\
\left(m m \cdot j^{-1}\right)\end{array}$ & $\begin{array}{c}R g \\
\left(W \cdot m^{-2}\right)\end{array}$ & $\begin{array}{c}I g \\
\left(I \cdot j^{-1}\right)\end{array}$ & $\begin{array}{c}I \\
\left(m m \cdot j^{-1}\right)\end{array}$ & $\begin{array}{c}C S_{i} \\
\left(m m \cdot j^{-I}\right)\end{array}$ & $\begin{array}{c}I+C S_{i} \\
\left(m m \cdot j^{-1}\right)\end{array}$ & $\begin{array}{c}C S_{n i} \\
\left(m m \cdot j^{-I}\right)\end{array}$ \\
\hline $8 / 8$ & 5,50 & 463,8 & 61,1 & 2,91 & 0,22 & 3,13 & 0,34 \\
$9 / 8$ & 6,70 & 492,0 & 61,1 & 2,91 & 0,22 & 3,13 & 0,33 \\
$10 / 8$ & 3,70 & 322,0 & 61,1 & 2,91 & 0,22 & 3,13 & 0,32 \\
$11 / 8$ & 5,00 & 467,2 & 60,7 & 2,89 & 0,21 & 3,10 & 0,31 \\
$12 / 8$ & 5,30 & 480,3 & 60,7 & 2,89 & 0,21 & 3,10 & 0,31 \\
$13 / 8$ & 4,80 & 476,6 & 60,7 & 2,89 & 0,21 & 3,10 & 0,30 \\
$14 / 8$ & 2,70 & 243,5 & 60,7 & 2,89 & 0,21 & 3,10 & 0,29 \\
$15 / 8$ & 4,70 & 459,1 & 60,7 & 2,89 & 0,20 & 3,09 & 0,28 \\
$16 / 8$ & 4,80 & 449,8 & 71,0 & 3,38 & 0,20 & 3,58 & 0,27 \\
$17 / 8$ & 4,20 & 395,8 & 48,9 & 2,33 & 0,20 & 2,53 & 0,26 \\
$18 / 8$ & 6,20 & 491,3 & 71,2 & 3,39 & 0,20 & 3,59 & 0,26 \\
$19 / 8$ & 4,60 & 469,6 & 57,1 & 2,72 & 0,19 & 2,91 & 0,25 \\
$20 / 8$ & 4,60 & 441,5 & 57,1 & 2,72 & 0,19 & 2,91 & 0,24 \\
$21 / 8$ & 4,50 & 438,1 & 66,4 & 3,16 & 0,19 & 3,35 & 0,23 \\
MOY. & 4,59 & 434,6 & 61,3 & 2,92 & 0,21 & 3,14 & 0,28 \\
$\sigma$ & 0,90 & 70,8 & 5,61 & 0,27 & 0,01 & 0,30 & 0,03 \\
\hline
\end{tabular}

$\mathrm{I}=$ irrigation journalière $; \mathrm{Ig}=$ apport d'eau par goutteur, $\mathrm{CS}_{\mathrm{i}}$ et $\mathrm{CS}_{\mathrm{ni}}=$ contribution du sol dans les traitements irrigué et non irrigué.

à pas de temps rapprochés, ont montré que les mesures à $30 \mathrm{~cm}$ du point de gouttage reflétaient parfaitement la situation dans le bulbe (Isbérie, 1992). L'étalonnage neutronique est de type gravimétrique (Normand, 1974). Les mesures sont faites tous les $10 \mathrm{~cm}$, de 15 à $275 \mathrm{~cm}$ de profondeur. Le pas de temps de mesure est de $15 \mathrm{j}$ pour la période du 14 avril au 31 octobre 1989, la précision des mesures rendant inutile une plus grande fréquence d'étude.

Les variations du stock hydrique entre chaque date de mesure sont calculées dans les deux sites, arbre irrigué et arbre non irrigué, sur une profondeur de $280 \mathrm{~cm}$, limite imposée par la profondeur du système de mesure. Leur suivi permet l'établissement des bilans de l'eau dans le sol et l'évaluation de l'importance des réserves naturelles du sol qui contribuent à l'alimentation hydrique des deux arbres (contribution du sol : CS). Les remontées capillaires, de la profondeur du sol vers la couche $280 \mathrm{~cm}$, sont objectivement impossibles à apprécier. Cependant elles peuvent être considérées comme nulles le sol n'ayant pas été réhumecté en profondeur pendant l'hiver précédent (Isbérie, 1992). On a négligé également l'évaporation à la surface du sol nu du fait d'une part du taux de couverture du sol par la frondaison et d'autre part de la très forte dessiccation de la surface du sol en bordure du verger.

Afin d'assurer l'extrapolation des mesures faites sur les tubes du rang à toute la surface cor- respondante à un arbre, des tubes supplémentaires avaient été antérieurement placés et suivis dans l'inter-rang à des distances équivalentes. Les bonnes corrélations trouvées entre les mesures effectuées sur le rang et sur 1'inter-rang, notamment au niveau des deux tubes les plus éloignés, ont permis de calculer une surface attribuable à chaque profil hydrique élémentaire établi sur le rang (Isbérie, 1992). Cette extrapolation a permis de calculer la contribution du sol à l'alimentation hydrique de chaque arbre pour chaque période comprise entre deux mesures. Quant à la contribution journalière du sol, elle est déduite de l'évolution générale de la contribution du sol mesurée sur trois périodes ; $(18 / 7-25 / 7 ; 25 / 7-17 / 8,17 / 8-6 / 9)$ encadrant la période ici analysée, les trois périodes étant par ailleurs dépourvues de précipitations notables au sol. Les valeurs de CS estimées (Ibérie, 1992) sont données au tableau I.

Le suivi des potentiels de l'eau du sol, nécessaire à la détermination du sens des flux de l'eau dans le sol et à la mise en évidence de l'absence de drainages profonds éventuels, notamment sous les points de gouttage, est assuré par tensiométrie. Seul le site irrigué est équipé de tensiomètres. Les mesures sont obtenues, entre 30 et $150 \mathrm{~cm}$ de profondeur, à partir de onze tensiomètres de marque Irrometer, équipés de manomètre Bourdon, situés dans et hors de la zone humectée par le goutteur (fig $1 \mathrm{~A}$ et $\mathrm{C}$ ). La mesure des potentiels est journalière. Leur précision est estimée à $\pm 1 \mathrm{kPa}$. 


\section{Quantification des transferts de sève brute dans les arbres}

La mesure des flux de sève brute est réalisée par la méthode thermique du flux radial (Granier, 1985 ; Cabibel et Do, 1991). Le flux de sève total U est égal au produit de la densité de flux Ju par la surface conductrice du bois $\mathrm{S}$ estimée dans le plan de la mesure.

$$
\mathrm{U}=\mathrm{Ju} . \mathrm{S}
$$

Ju étant exprimée en $\mathrm{m}_{\mathrm{s}} \mathrm{s}^{-1}$ et $\mathrm{S}$ en $\mathrm{m}^{2}$, U s'exprime en $\mathrm{m}^{3} . \mathrm{s}^{-1}$. La densité de flux Ju est déduite de la relation d'étalonnage la liant à l'index de flux $\mathrm{K}$, lui-même déterminé par le suivi des différences de température existant au niveau des deux sondes du capteur. La variable $\mathrm{K}$, sans dimension, a pour expression :

$$
\begin{aligned}
\mathrm{K} & =\frac{(\mathrm{To}-\mathrm{Tbo})-(\mathrm{Tu}-\mathrm{Tb})}{(\mathrm{Tu}-\mathrm{Tb})} \\
& =\frac{\delta \mathrm{To}-\delta \mathrm{Tu}}{\delta \mathrm{Tu}}=\frac{\delta \mathrm{To}}{\delta \mathrm{Tu}}-1
\end{aligned}
$$

où To et Tbo sont les températures de la sonde chaude et du milieu bois-sève à flux nul, Tu et $\mathrm{Tb}$ les températures de la sonde chaude et du milieu bois-sève à flux $U, \delta$ To la différence maximale de température entre sondes à flux nul et $\delta \mathrm{Tu}$ la différence de température à flux U. Pour une séquence de mesure de $n$ jours, l'estimation du terme $\delta$ To est réalisée, pour chaque sonde, en prenant l'enveloppe supérieure de la succession des maxima nocturnes de la période concernée. La relation $\mathrm{K}(\mathrm{Ju})$, de type $\mathrm{K}=\alpha \cdot \mathrm{Ju}^{\beta}$, est obtenue par ajustement non linéaire des couples de valeurs de $\mathrm{K}$ et de Ju, lors d'étalonnages au laboratoire (Granier, 1985 ; Do, 1987). La surface $S$ est déduite pour chaque capteur de la mesure de l'épaisseur de bois conducteur présente localement. Cette estimation est obtenue, pour chaque fluxmètre, par carottages dans le tronc, en fin de campagne de mesure, dans le plan des sondes. Dans le cas étudié, seule une couronne extérieure de bois ayant été trouvée conductrice, l'expression du flux est :

$$
\mathrm{U}=[\mathrm{K} / \alpha]^{1 / 3} \cdot \pi \cdot\left(\mathrm{R}^{2}-\mathrm{r}^{2}\right)
$$

où $\mathrm{R}$ et $\mathrm{r}$ sont respectivement le rayon total du bois du tronc et le rayon de bois colmaté non conducteur, $\alpha$ et $\beta$ les coefficients d'ajustement non linéaire de la relation $K(\mathrm{Ju})$.

Quatre fluxmètres sont placés dans l'arbre I, selon les quatre orientations cardinales (FN, FE, FS, FO) ; deux fluxmètres sont placés dans l'arbre NI, en est et en ouest (fe et fo) (fig 1D). Les sondes, de $2 \mathrm{~cm}$ de long, sont distantes de $10 \mathrm{~cm}$. Le long du tronc, elles sont implantées sur des génératrices légèrement décalées latéralement de façon à ne pas interrompre les vaisseaux conducteurs conduisant la sève jusqu'à la sonde chaude supérieure. Celle-ci est située à $30 \mathrm{~cm}$ du sol. Les mesures sont faites avec un pas de temps de 30 min. La prise de mesure est gérée par ordinateur. Les flux élémentaires au niveau de chaque capteur sont calculés en fonction des surfaces conductrices qui leurs sont associées. Des secteurs de 90 et $180^{\circ}$ sont respectivement associés aux capteurs des arbres I et NI. Les flux totaux traversant un tronc sont calculés par sommation des flux élémentaires déterminés sur chacun des fluxmètres implantés dans le tronc Les flux traversant la section du tronc sont rapportés, quand exprimés en millimètre, à une surface au sol de $42 \mathrm{~m}^{2}$ correspondant à l'espacement $6 \times 7 \mathrm{~m}$ entre les arbres. Les flux élémentaires, estimés au niveau de chaque fluxmètre, sont rapportés, quand exprimés en millimètre, à une surface au sol de $10,5 \mathrm{~m}^{2}$ dans le cas de l'arbre irrigué (quatre fluxmètres) et de $21 \mathrm{~m}^{2}$ dans le cas de l'arbre non irrigué (deux fluxmètres). Les flux horaires et journaliers sont respectivement donnés avec une incertitude relative inférieure à 15 et $10 \%$ (Cabibel et Do, 1991).

\section{Mesures du diamètre des branches et de l'amplitude de la contraction diurne (ACD)}

Elles sont estimées selon la méthode proposée par Huguet (1985), à l'aide de capteurs de déplacement linéaires. Ils sont connectés à un système d'acquisition et de traitement de données Pépista (Copa-Technologie), qui effectue les mesures chaque demi-heure, avec une précision de l'ordre de $10 \mu \mathrm{m}$, et calcule les valeurs journalières de l'ACD et de la croissance. Deux capteurs sont installés par arbre, sur des branches de diamètre comparable (4 à $5 \mathrm{~cm}$ ), d'orientation et d'inclinaison identiques. Les capteurs sont respectivement en position ouest (C1) et sud-est (C2) dans l'arbre $\mathrm{I}$, en est (C3) et ouest (C4) dans l'arbre NI (fig 1D).

\section{RÉSULTATS}

L'expérimentation est conduite du 8 au 21 août 1989, période durant laquelle l'ensemble des mesures dans le sol et sur l'arbre est réalisé. En l'absence de précipitations significatives, une bonne approche dans l'évaluation des bilans de 
l'eau dans le sol et de la contribution propre du sol (CS) à l'alimentation hydrique des arbres est possible : l'absence de ruissellement et de drainage permet en effet la simplification dans l'écriture des bilans hydriques dans le sol. Les résultats sont donnés en heure locale.

\section{Transferts de sève brute dans les arbres}

Ils se caractérisent par une hétérogénéité qui peut être très importante : la densité de flux de sève est très variable selon le secteur du tronc et les flux sont beaucoup plus faibles dans l'arbre non irrigué. Pour toute la séquence de mesures, les flux totaux et les flux estimés au niveau de chaque fluxmètre sont donnés en $\mathrm{mm} \cdot \mathrm{J}^{-1}$ au tableau II.

\section{Variabilité des flux dans la section du tronc}

Les cinétiques de flux horaires sont présentées en figure 2, pour la période du 8 au 14 août, période représentative de l'ensemble de la période étudiée. Leur analyse montre que, pour les deux traitements, l'importance des flux et l'allure de leurs cinétiques nycthémérales sont fonction de la génératrice de mesure dans le tronc. L'hétérogénéité de répartition des flux, dans la section du tronc des arbres I et NI, est donc générale.
En présence d'irrigation, cette hétérogénéité devient très importante. Pour les deux capteurs présentant des flux extrêmes, les capteurs FO et FS, capteurs qui correspondent à des faisceaux racinaires supposés s'alimenter respectivement en zones irriguée et non irriguée localement, les rapports de flux varient, à l'échelle horaire de minima compris entre 6 et 7 (11-12 heures) à des maxima compris entre 29 et 57 (19 et 20 heures). Ce rapport crô̂t systématiquement après le déclenchement de l'irrigation et pour une même dose journalière d'irrigation avec l'accroissement de la demande climatique instantanée.

Par ailleurs si la cinétique des flux en FO apparaît synchrone de celle de la contrainte climatique (fig 2A), elle n'est pas synchrone de celle-ci en FS. En FO les flux apparaissent avec la demande climatique, croissent avec elle, passent par un maximum au maximum du $\mathrm{Rg}$ puis décroissent comme décroît l'ETP. Seule différence, l'existence de flux tardifs générés par l'irrigation dès 17 heures. En FE, si les flux apparaissent aussi avec la contrainte climatique, ceux-ci passent par un maximum bien avant le maximum du Rg (10 heures) pour décroître rapidement et très fortement alors même que la contrainte climatique continue de croître. Sur cette génératrice les cinétiques obtenues font apparaître une faible disponibilité de l'eau dès l'apparition d'un rayonnement global horaire élevé.

Tableau II. Flux journaliers mesurés sur l'ensemble des génératrices de mesure et dans les troncs des arbres selon le traitement hydrique appliqué.

\begin{tabular}{|c|c|c|c|c|c|c|c|c|}
\hline \multirow[t]{3}{*}{ Date } & \multicolumn{8}{|c|}{ Flux de sève $\left(\mathrm{mm} \cdot \mathrm{j}^{-l}\right)$} \\
\hline & \multicolumn{4}{|c|}{ Capteurs I } & \multicolumn{2}{|c|}{ Capteurs NI } & \multicolumn{2}{|c|}{ Troncs } \\
\hline & Nord & Est & Sud & Ouest & $E s t$ & Ouest & $I$ & $N I$ \\
\hline $8 / 8$ & 2,81 & 0,43 & 0,40 & 6,06 & 0,38 & 0,45 & 2,42 & 0,41 \\
\hline $9 / 8$ & 2,58 & 0,54 & 0,48 & 5,98 & 0,39 & 0,45 & 2,40 & 0,42 \\
\hline $10 / 8$ & 2,42 & 0,58 & 0,51 & 5,51 & 0,31 & 0,42 & 2,26 & 0,36 \\
\hline $11 / 8$ & 2,99 & 0,40 & 0,42 & 5,73 & 0,37 & 0,44 & 2,39 & 0,40 \\
\hline $12 / 8$ & 3,30 & 0,45 & 0,41 & 6,03 & 0,37 & 0,46 & 2,55 & 0,41 \\
\hline $13 / 8$ & 3,00 & 0,42 & 042 & 5,60 & 0,31 & 0,42 & 2,36 & 0,37 \\
\hline $14 / 8$ & 2,40 & 0,48 & 0,46 & 4,59 & 0,27 & 0,30 & 1,98 & 0,28 \\
\hline $15 / 8$ & 3,33 & 0,38 & 0,39 & 5,47 & 0,33 & 0,43 & 2,39 & 0,38 \\
\hline $16 / 8$ & 3,61 & 0,43 & 0,37 & 6,03 & 0,33 & 0,42 & 2,61 & 0,38 \\
\hline $17 / 8$ & 3,21 & 0,58 & 0,46 & 5,26 & 0,31 & 0,35 & 2,38 & 0,33 \\
\hline $18 / 8$ & $3,5 \mathrm{I}$ & 0,54 & 0,47 & 5,75 & 0,31 & 0,40 & 2,57 & 0,36 \\
\hline $19 / 8$ & 3,63 & 0,51 & 0,45 & 5,94 & 0,29 & 0,42 & 2,64 & 0,35 \\
\hline $20 / 8$ & 3,39 & 0,49 & 0,41 & 5,45 & 0,31 & 0,37 & 2,44 & 0,34 \\
\hline $21 / 8$ & 3,28 & 0,43 & 0,39 & 5,54 & 0,33 & 0,38 & 2,41 & 0,35 \\
\hline MOY. & 3,09 & 0,47 & 0,43 & 5,64 & 0,33 & 0,41 & 2,41 & 0,36 \\
\hline$\sigma$ & 0,38 & 0,07 & 0,04 & 0,40 & 0,04 & 0,04 & 0,16 & 0,04 \\
\hline
\end{tabular}




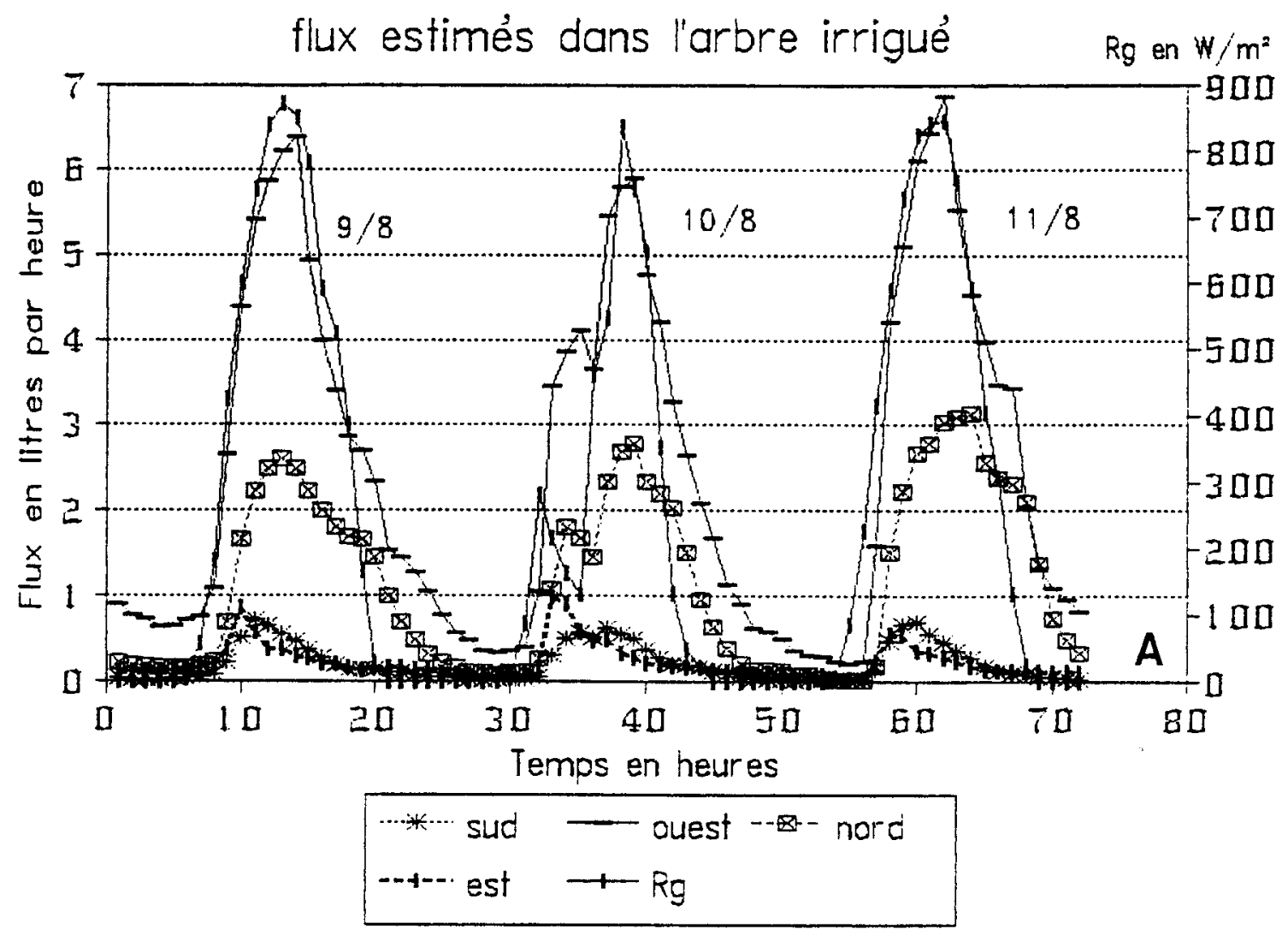

Flux estimés dans l'arbre non irrigué $\mathrm{Rg}$ en $\mathrm{W} / \mathrm{m}^{\text {" }}$

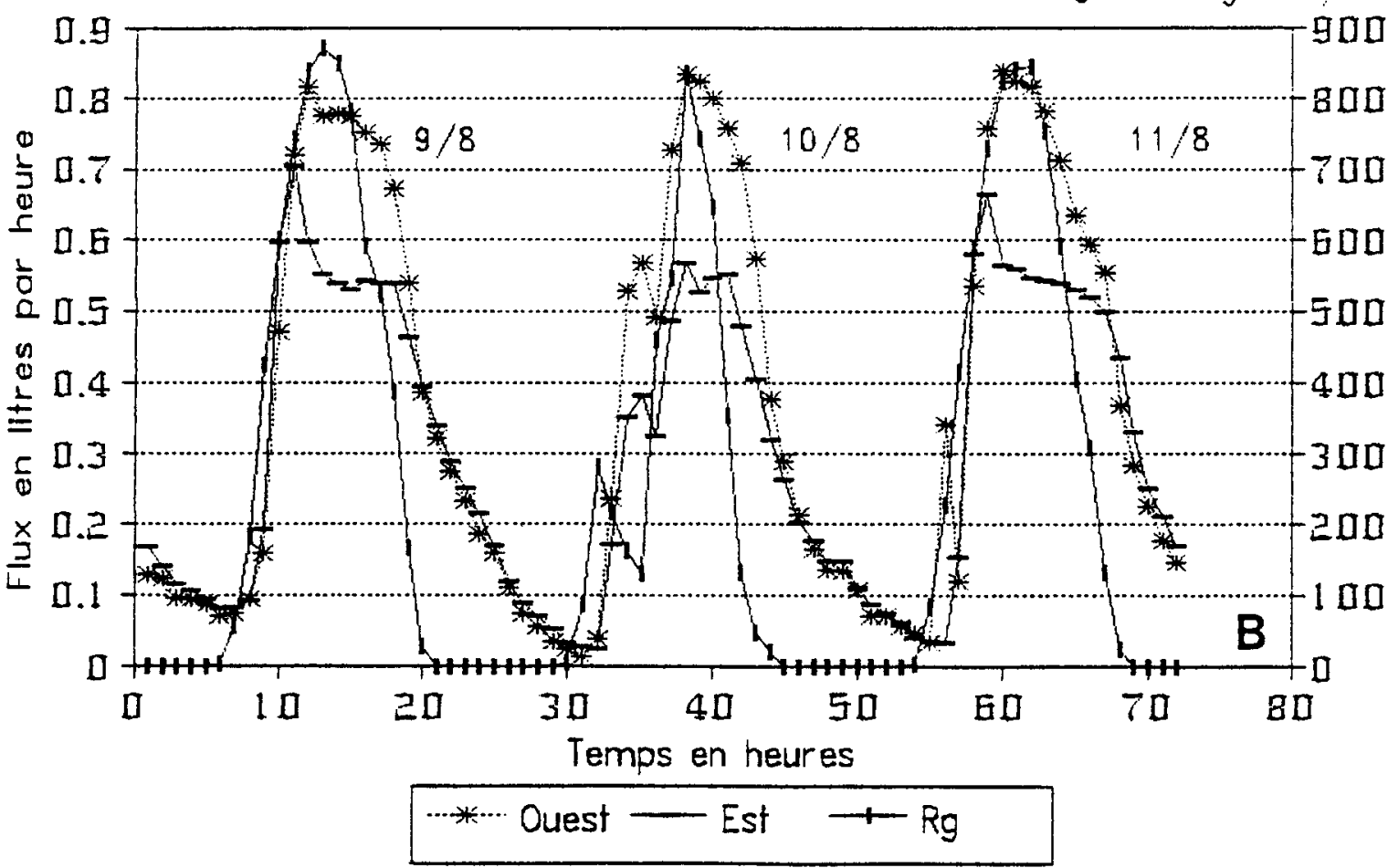

Fig 2. Cinétiques des flux horaires de sève brute dans les troncs selon l'orientation des fluxmètres : arbre irrigué (A), arbre non irrigué (B). 
À l'échelle journalière le rapport des flux FO/FS varie de 9,9 à 16,4 . Sa valeur moyenne est de 13,2 $\pm 1,7$. Cette valeur est très proche de celle trouvée pour le rapport I/CS, apport d'eau par irrigation / contribution naturelle du sol, qui est égale à 13,9 (tableau II). La comparaison des flux journaliers estimés sur les quatre génératrices du tronc, en fonction des doses d'eau journalières disponibles dans le sol (irrigation I + contribution naturelle du sol CSi) (figure 3), montre que, pour les $14 \mathrm{j}$ étudiés, les valeurs moyennes des flux estimées sont :

- relativement proches de celles de la Cs aux capteurs FE et FS ;

- proches de la quantité d'eau apportée par le goutteur au capteur FO ;

- en position intermédiaire au capteur FN.

L'hétérogénéité locale des flux de sève dans la section du tronc apparaît donc étroitement liée à l'hétérogénéité locale de l'alimentation hydrique au niveau du sol. Ce phénomène confirme l'existence de voies préférentielles pour les flux, voies reliant étroitement un faisceau racinaire au secteur de sol qui le contient d'une part et au secteur du tronc qui lui correspond d'autre part (Cabibel, 1994). Néanmoins la symétrie existant dans le sol en ce qui concerne la fourniture en eau (goutteur en est et en ouest) ne se retrouve pas pleinement à l'identique dans le tronc de l'arbre : des flux importants, qui croissent au déclenchement de l'apport d'eau, apparaissent en effet au capteur nord et non au capteur est, capteur auquel ces flux devraient être théoriquement attribués puisque à l'aplomb de la zone du goutteur est (fig 1A). Cette distorsion peut être le fait d'une vérification locale seulement partielle de l'hypothèse de continuité directionnelle des faisceaux de racines primaires de l'arbre. Lors de l'arrachage d'arbres de cette parcelle, il a été en effet trouvé un petit nombre de racines primaires ne conservant pas leur direction initiale. Celles-ci avaient tourné autour du tronc. Ce vrillage peut être le résultat d'une torsion d'une partie du système racinaire lors de sa mise en place à la plantation suivi d'un tropisme vers les points de gouttage pour les racines les plus superficielles, phénomène déjà constaté sur pommier (Cabibel, 94).

En l'absence d'irrigation les rapports des flux fo/fe, mesurés à l'échelle horaire sur les deux génératrices du tronc de l'arbre NI demeurent relativement faibles. Ils sont maxima aux heures de plus forte demande climatique (fig 2B). Ils varient de 0,5 à 3,0 . Les cinétiques des flux ne sont ni synchrones entre elles, ni synchrones de celle de la contrainte climatique. Elles sont caractéristiques d'un stress hydrique important dans l'arbre, dés l'apparition d'une ETP horaire importante. Sur les deux génératrice de mesures, les flux, par ailleurs faibles, présentent très tôt des maxima, bien avant les maxima du $\mathrm{Rg}$, puis décroissent ensuite très rapidement jusqu'à leurs valeurs minimales. Ces phénomènes sont plus marqués et se manifestent plus tôt en Fe qu'en Fo. Ces différences de comportement dans l'arbre peuvent être reliées à son environnement : placé en bordure est du verger, l'arbre est soumis à une demande climatique plus

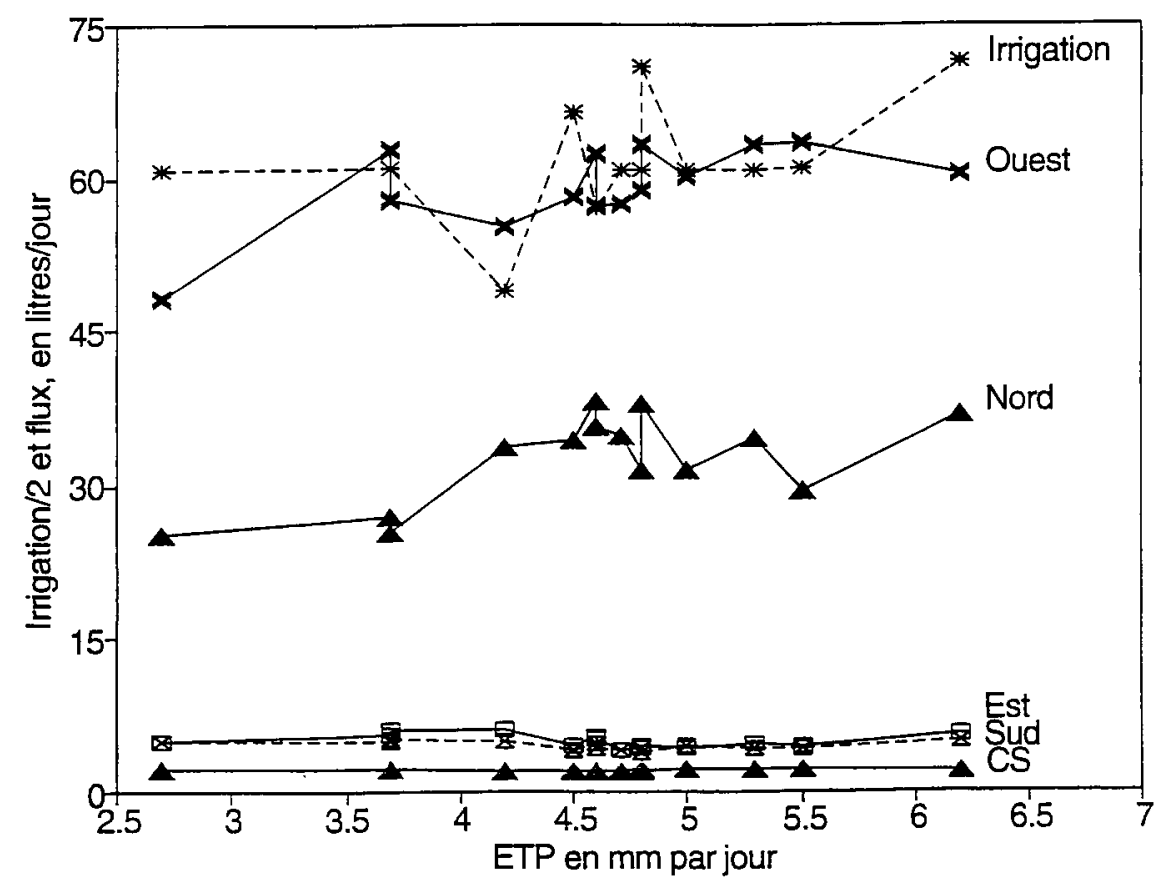

Fig 3. Évolution de l'apport d'eau par goutteur, de la contribution naturelle du sol (CS) et des flux de sève brute obtenus sur les quatre fluxmètres de l'arbre irrigué en fonction des ETP. 
matinale et importante en est qu'en ouest ; bordé en est par un chemin soumis à une évaporation intense du fait d'une non couverture foliaire et de l'absence de précipitations, le faisceau racinaire est explore un profil de sol plus asséché qu'en ouest. Les racines présentes dans ce secteur est du sol verraient en conséquence, leurs possibilités d'absorption hydrique plus rapidement limitées qu'en ouest face à une succession d'équilibres instantanés croissants entre l'intensité de la demande climatique et l'aptitude du sol à maintenir une disponibilité suffisamment élevée de l'eau à l'interface sol-racine, dès que la demande climatique devient forte. Inversement le faisceau racinaire ouest explore un profil de sol intraparcellaire, qui, peu soumis à l'évaporation du fait d'une couverture foliaire totale, demeurerait plus régulièrement humide sur toute sa profondeur. La disponibilité de l'eau du sol, bien que faible, y demeurerait plus grande et plus continue face à une demande climatique plus forte.

\section{Relations flux, alimentation hydrique des arbres, ETP}

La comparaison des flux moyens traversant les troncs dans la période allant du $8 / 8$ au $14 / 8$ (tableau II) et de l'alimentation hydrique moyenne des arbres durant la même période (tableau I), ces variables étant exprimées en millimètre, fait apparaître que ces flux représentent respectivement en moyenne 77 et $128 \%$ des alimentations hydriques correspondantes pour l'arbre irrigué et l'arbre non irrigué. Il y aurait donc déficit de prélèvement hydrique pour l'arbre I par rapport à l'irrigation bien que celle-ci ait été toujours très inférieure à la demande climatique, et surabsorption pour l'arbre NI par rapport à la contribution naturelle de sol estimée. L'évaporation dans la zone mouillée pouvant être considérée comme négligeable, les suivis tensiométriques n'ayant jamais mis en évidence de drainages profonds sous goutteur, les apports d'eau et les sections conductrices de bois conducteur ayant fait l'objet de contrôle rigoureux, les écarts constatés entre les flux mesurés et les alimentations hydriques peuvent être essentiellement imputés aux estimations de flux par la méthode thermique utilisée. Dans l'arbre I, le déficit d'absorption serait lié à une sous estimation des flux correspondants à des densités de flux élevées (DO, 1987); dans l'arbre NI, l'excès d'absorption hydrique serait lié à une surestimation des flux correspondants à des densités de flux très faibles (Do, 1987) et/ou à une sous-estimation des réserves en eau dans le sol liée à la faible impor- tance des différences trouvées lors de l'estimation des bilans de l'eau dans le sol. Cet arbre en bordure du verger peut avoir exploité, du fait d'une adaptation à des conditions de sécheresse depuis sa plantation, un volume de sol (surface, profondeur) supérieur à la référence adoptée pour le calcul de la CS. Néanmoins, si ces hypothèses étaient confirmées, les conclusions avancées précédemment, lors de l'analyse des flux élémentaires, sur l'existence de voies préférentielles pour les flux hydriques dans le système secteur du sol-faisceau racinaire contenu dans ce secteur de sol-secteur du tronc en liaison avec le faisceau racinaire se trouveraient renforcées.

Il n'existe pas, sous irrigation, de relation stricte entre l'alimentation hydrique $(\mathrm{I}+\mathrm{CSi})$ et l'amplitude des flux. Les résultats montrent seulement qu'aux plus fortes alimentations hydriques (16 et 18 août) correspondent généralement les flux les plus importants au jour de l'irrigation et des flux accrus le jour suivant Les flux de sève apparaissent donc essentiellement commandés par l'amplitude et l'évolution des quantités d'eau disponibles dans le sol. Cette conclusion est confirmée par l'analyse comparée des flux mesurés le 19 et le 20 août, jours où s'est exercée sur le verger la même demande climatique $(4,6 \mathrm{~mm})$ et où a été pratiquée la même irrigation $(2,72 \mathrm{~mm})$. L'amplitude plus importante du flux le 19 août doit résulter de l'accroissement des réserves en eau du sol, consécutif à la forte irrigation de la veille. Il en est de même le 17 août, jour à plus faible irrigation $(2,33 \mathrm{~mm})$ où le niveau relativement élevé des flux mesurés doit résulter de l'accroissement des quantités d'eau disponibles dans le sol du à la très forte irrigation du 16 août $(3,38 \mathrm{~mm})$.

Il n'existe pas de relation stricte entre l'importance des flux et le niveau de la contrainte climatique s'exerçant sur le verger. Celle-ci n'intervient que dans une très faible mesure. En présence d'irrigations équivalentes, les plus fortes ETP engendrent de faibles accroissements des flux et les plus faibles une légère diminution de ceux-ci. En présence d'irrigations, lesquelles sont en général restées très inférieures à la demande climatique, la transpiration de l'arbre irrigué s'est donc ajustée à la quantité d'eau apportée, la disponibilité des réserves naturelles du sol étant très faibles.

En traitement non irrigué, les flux, toujours très faibles, sont évidemment uniquement fonction de la contribution naturelle du sol et de sa disponibilité instantanée relative par rapport à l'amplitude de la contrainte climatique. 


\section{Microvariations des diamètres des branches}

On donne en figure $4 \mathrm{~A}$ et $\mathrm{B}$ les variations de diamètre des branches, exprimées en $\mu \mathrm{m}$, trouvées au niveau des quatre capteurs de déplacement étudiés. Les écarts horaires, exprimés en $\mu \mathrm{m} \cdot \mathrm{h}^{-1}$, sont donnés en figure $4 C$ et $D$. L'évolution de ces mêmes paramètres, moyennés pour les deux dendromètres situés sur un même arbre, est donnée en figure $5 \mathrm{~A}$ et $\mathrm{B}$.

\section{Comportement relatif des capteurs situés dans un même arbre}

Dans l'arbre irrigué, les dendromètres ( $\mathrm{C} 1$ et $\mathrm{C} 2)$ ont des comportements très proches, quelle que soit l'échelle de temps d'observation (figure 4A et $\mathrm{C}$ ) ; leurs cinétiques sont synchrones et leurs amplitudes de déplacement sont sensiblement égales. Dans les deux branches, les réserves en eau y sont donc mobilisées et reconstituées de façon pratiquement identique en quantité et dans le temps. Le comportement hydrique de l'arbre est donc homogène. Dans l'arbre NI, au contraire, l'évolution des variations des diamètres révèle un comportement très différencié des réserves en eau propres de l'arbre, selon l'orientation de la branche étudiée (figure 4B et D). La participation de la branche est (C3) à l'évapotranspiration apparaît toujours plus tôt et est généralement moins importante et moins continue dans le temps. La reconstitution des réserves mobilisées y apparaît également plus tôt et est beaucoup plus irrégulière qu'en ouest $(\mathrm{C} 4)$. À l'échelle journalière la perte en diamètre, qui affecte les deux branches, est relativement moins importante en $\mathrm{C} 4$, notamment les jours de plus faible demande climatique. En ouest, les réserves en eau de l'arbre mobilisées sont donc reconstituées à un niveau plus élevé qu'en est sous l'effet de flux racinaires plus élevés. Il semblerait donc que cet arbre fortement stressé n'arrive pas à homogénéiser aussi bien que l'arbre irrigué son état hydrique au niveau de la couronne

\section{Comportement relatif moyen des différents capteurs en fonction de l'alimentation hydrique}

L'analyse comparée des mesures de variations de diamètre montre que les cinétiques de contraction et d'expansion sont sous la dépendance générale de la contrainte climatique subie dans les deux traitements. Néanmoins le phénomène étudié (contraction ou expansion) n'est pas, dans les deux cas, synchrone et est d'intensité inégale (fig 5A). Leur apparition dans le temps et leur intensité apparaissent donc également en fonction du traitement hydrique appliqué à l'arbre. L'évolution comparée des variations des diamètres (fig 5B) montrent qu'en l'absence d'irrigation :

- les réserves en eau propres de l'arbre sont toujours mobilisées plus tôt (décalage de 1 à $2 \mathrm{~h}$ ) du fait d'une moindre disponibilité de l'eau dans les profils de sol explorés par les faisceaux racinaires,

- l'intensité initiale de cette participation au courant de transpiration est quantitativement plus importante,

- la mobilisation des réserves de l'arbre s'effectue sur un temps plus court, et s'arrête alors même que la demande climatique croît. Ces observations peuvent être le fait d'une régulation stomatique précoce, conséquence de stress hydriques qui se développent dans l'arbre dès l'apparition de contraintes climatiques importantes. La régulation stomatique aurait pour résultats d'induire successivement une réduction des flux évapotranspiratoires et donc de la sollicitation des réserves propres en eau de l'arbre, puis l'utilisation non totale mais préférentielle des flux racinaires encore existants à la régénération des réserves en eau de l'arbre précédemment mobilisées

L'évolution des variations des diamètres (fig 5A) montre que, sous irrigation, existe soit une permanence du diamètre initial, soit, à plus long terme, un faible accroissement de celui-ci. L'irrigation satisferait donc seulement au besoin minimum de l'arbre. Inversement, en l'absence d'irrigation, la reconstitution de ces réserves n'étant que très partielle, les branches ne récupèrent pas, à long terme, leurs diamètres initiaux. Ils présentent une décroissance continue. Il existe donc sous ce traitement un déficit très marqué d'alimentation en eau, déficit qui augmente comme diminue, dans le temps, la contribution hydrique du sol à son alimentation.

\section{Relations entre variations de diamètre et flux de sève}

L'évolution moyenne du diamètre des branches en fonction des flux de sève brute traversant les troncs I et NI, lors d'un jour de moyenne ETP (5 $\mathrm{mm}$ ) est donnée en figure 6. L'évolution comparée des flux de sève brute, des diamètres moyens des branches et du rayonnement global, un jour d'ETP faible $(2,7 \mathrm{~mm})$, présentant des passages nuageux, est donnée en figure 7 . Ces cinétiques permettent 


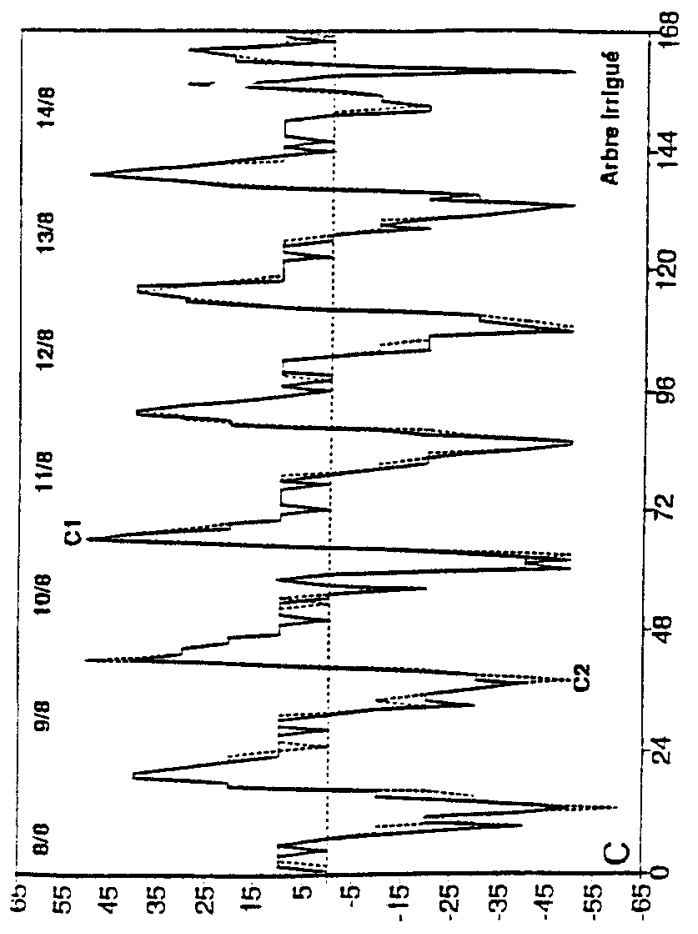

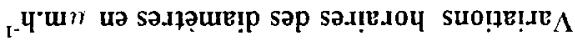

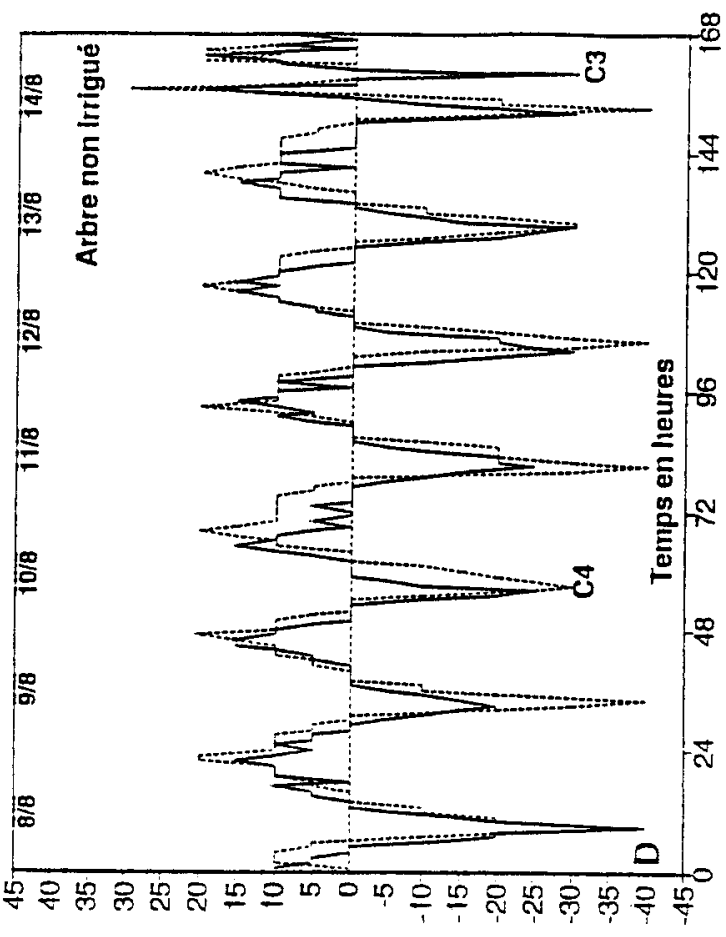

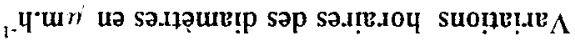

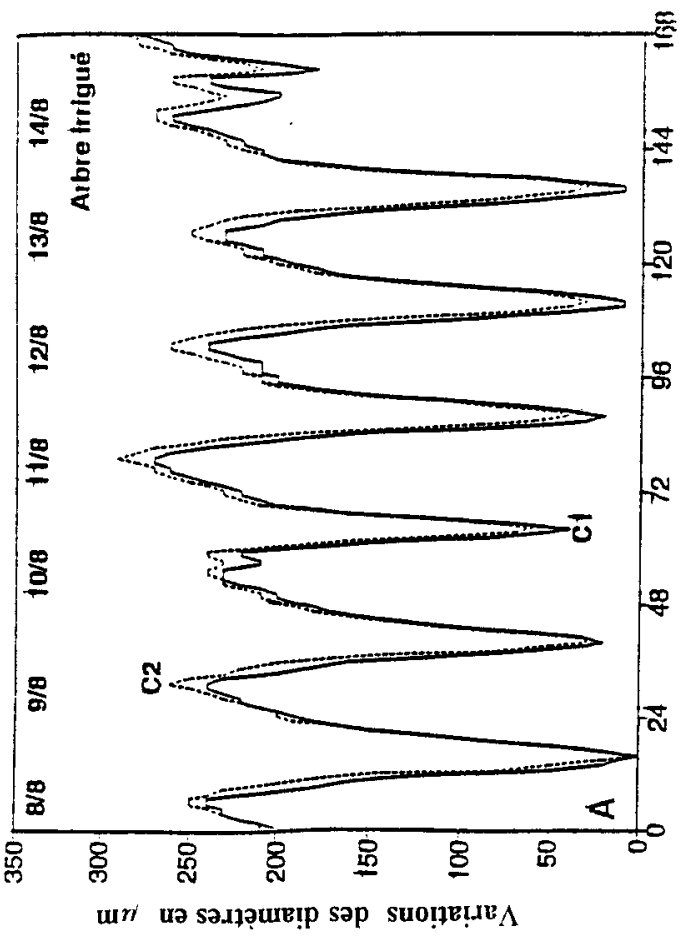

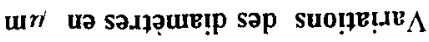

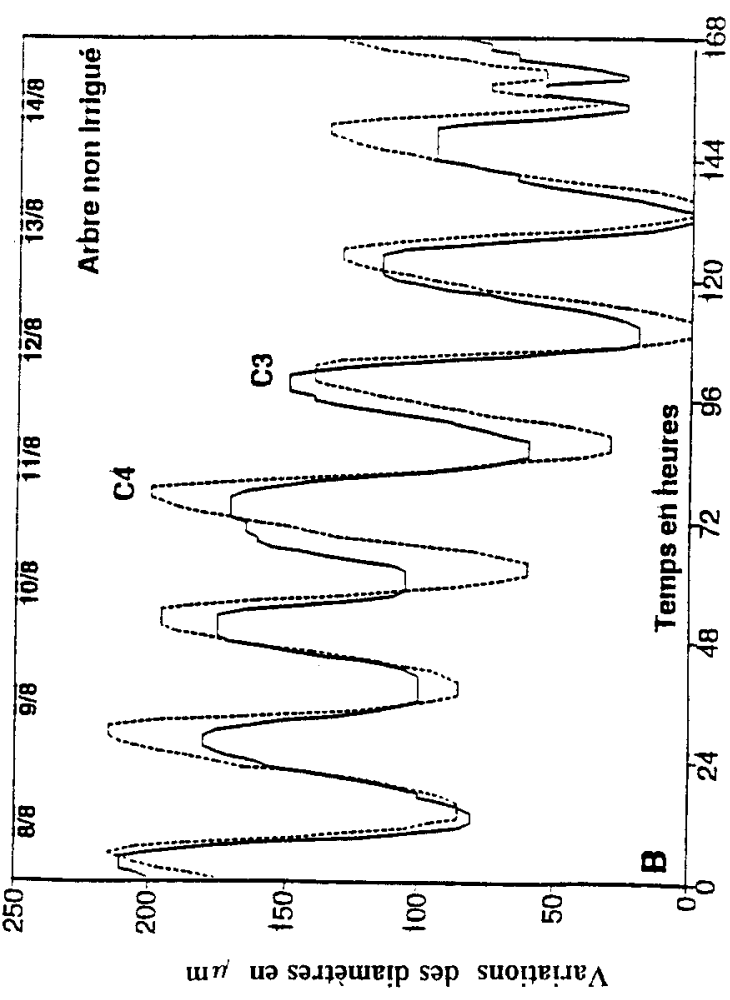



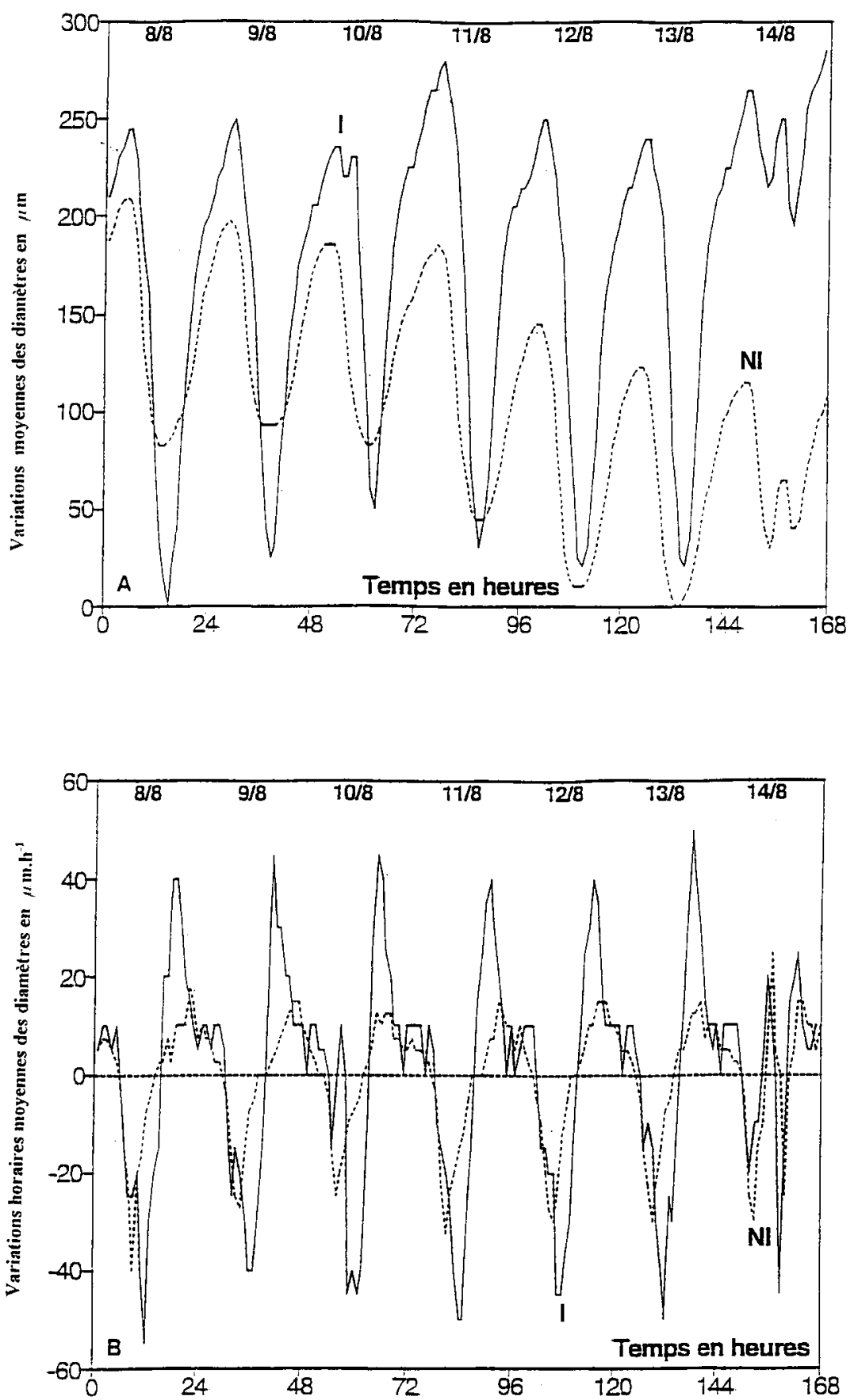

Fig 5. Évolution relative moyenne des variations du diamètre des branches en fonction de l'alimentation hydrique de l'arbre : variations brutes en $\mu \mathrm{m}(\mathrm{A})$, variations horaires en $\mu \mathrm{m} \cdot \mathrm{h}^{-1}(\mathrm{~B})$.

de juger, selon les différences d'alimentation en eau des arbres, la participation relative des deux sources d'eau, extraction racinaire dans le sol et réserves en eau propres de l'arbre, au courant de transpiration, face à une demande climatique donnée.

\section{Cas d'une ETP moyenne}

En présence d'irrigation (fig 6), les parties ligneuses ne contribuent pas au flux transpiratoire entre 5 et 8 heures, la diminution des diamètres des branches étant nulle. Inversement, avec l'augmentation de l'ETP, les réserves en eau des parties ligneuses de l'arbre participent au courant de transpiration entre 8 et 17 heures, en fonction du différentiel transpiration-flux de sève. Le maximum de contraction horaire est atteint à 13 heures lorsque les flux commencent à diminuer. La contraction maximale est atteinte à 17 heures alors que les flux passent par un premier minimum. Par la suite le diamètre des branches augmente de façon importante sous l'effet combiné des flux racinaires, notamment accrus par l'irrigation, et de la diminution de la demande climatique. Il 


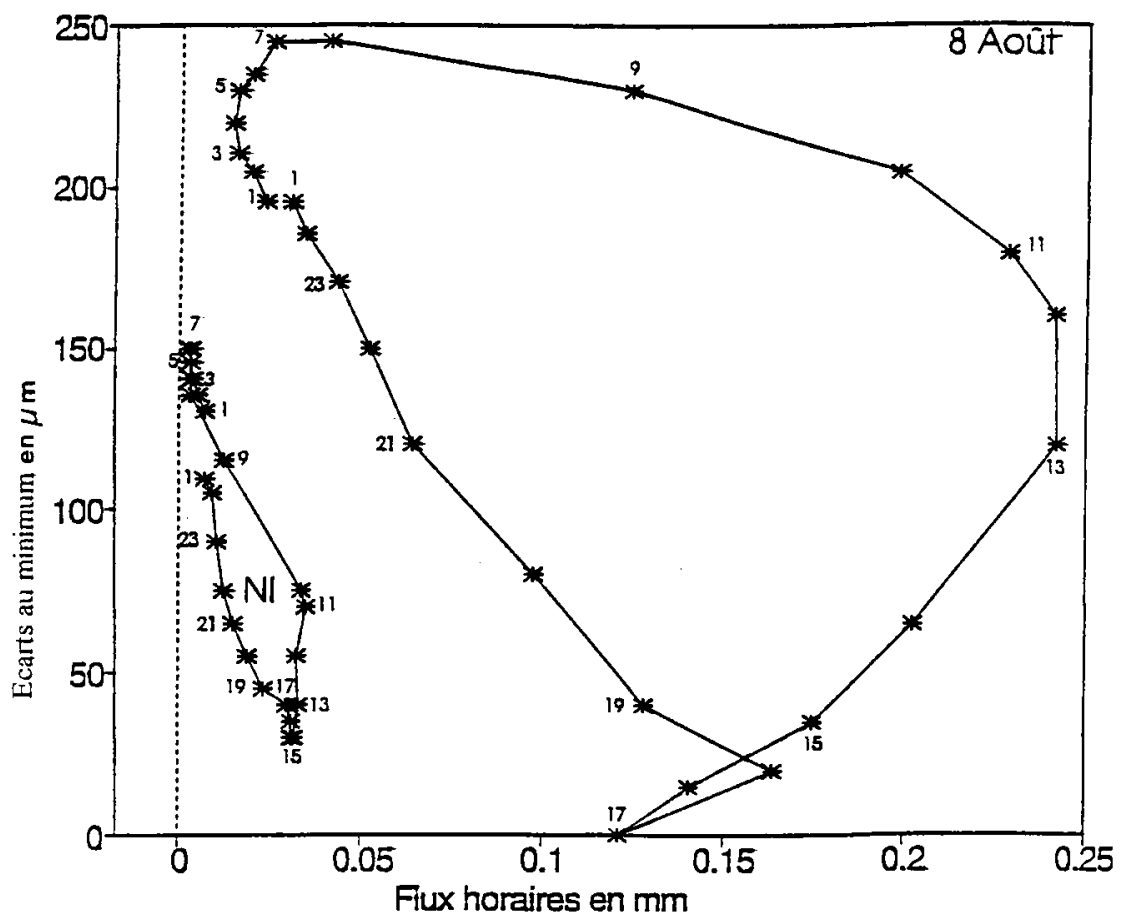

Fig 6. Relation entre les variations de diamètre des branches par rapport au minimum en $\mu \mathrm{m}$ et les flux horaires de sève brute.
Fig 7. Évolution comparée des flux de sève, du rayonnement global ( $\mathrm{Rg})$ et des variations moyennes du diamètre des branches dans le temps (FI et FNI : flux de sève dans l'arbre irrigué et non irrigué ; Depl et DepNI : variations du diamètre par rapport au minimum en $\mu \mathrm{m}$ ).

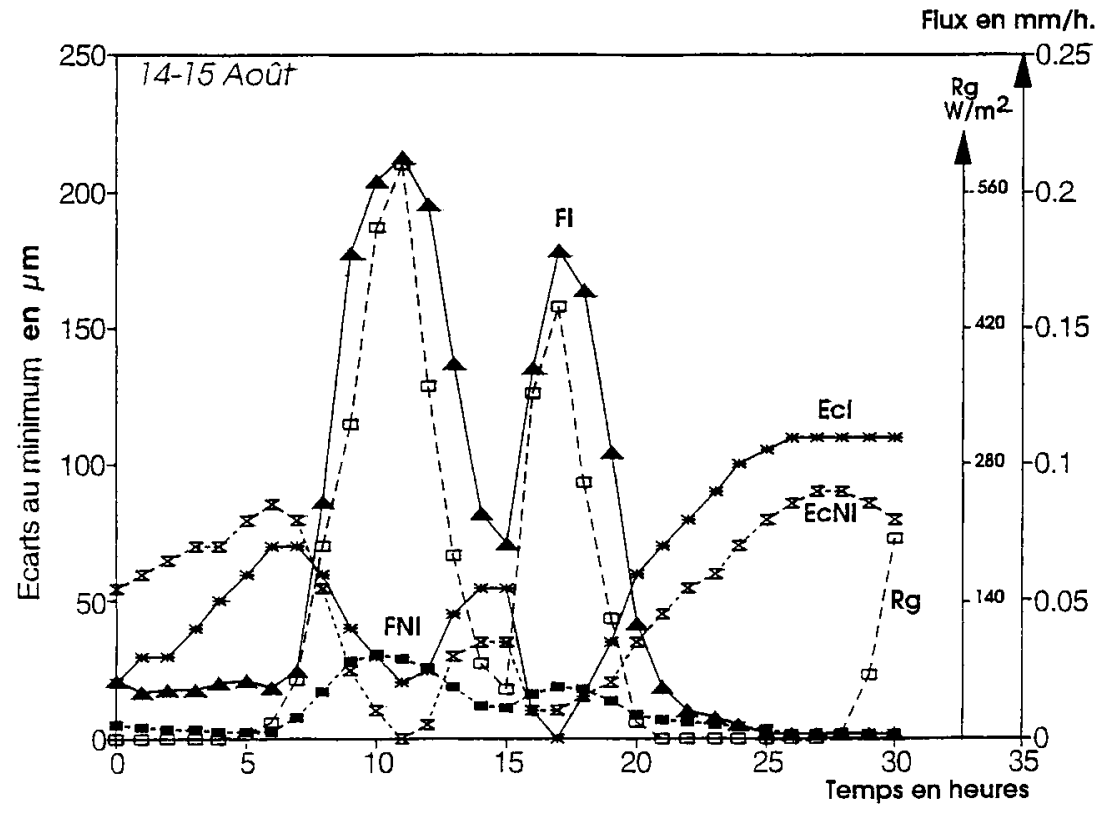

réatteint sa valeur initiale sous l'effet des flux nocturnes. En l'absence d'irrigation, la participation des réserves en eau des parties ligneuses aériennes de l'arbre au courant de transpiration apparaît plus tôt (7 heures) et est relativement intense durant toute la période d'accroissement des flux. La contraction horaire maximale est atteinte entre 10 et 11 heures. Les flux racinaires étant alors stabilisés à un niveau trop faible pour satisfaire à l'aug- mentation de la demande climatique, la perte en diamètre des branches continue de croître jusqu'à 14 heures où elle atteint sa valeur cumulée maximale.

L'accroissement de diamètre n'est effectif qu'à 16 heures. Avec la diminution de l'ETP, le diamètre croît à nouveau, les flux tardifs et nocturnes, très faibles, ne permettant qu'une régénération partielle des réserves en eau de l'arbre. 
Un indice de la participation relative des deux sources d'eau, interne et externe, au courant de transpiration, dont la gamme de variation sera spécifique du verger, peut s'exprimer, dans un intervalle de temps donné, par le rapport variation de diamètre des branches/flux de sève, ces variables étant mesurées dans l'intervalle de temps considéré. Ces rapports sont respectivement égaux à -680 et $-166 \mu \mathrm{m} \cdot \mathrm{mm}^{-1}$ en NI et en I, dans la période de contraction des diamètres. Ces valeurs confirment une participation relative beaucoup plus active des réserves de l'arbre NI à l'évapotranspiration. Inversement, dans la période d'expansion des diamètres, ils sont respectivement égaux à 475 et $251 \mu \mathrm{m} \cdot \mathrm{mm}^{-1}$. La valeur élevée de ce rapport en NI montre que les flux de sève existants participent, en présence d'une régulation stomatique, intensément à la régénération des réserves en eau de l'arbre. La faiblesse des flux tardifs et nocturnes, limités par la très faible disponibilité en eau du sol, constitue le facteur limitant à une régénération totale des réserves en eau propres de l'arbre. Inversement, sous irrigation, une valeur plus élevée de ce rapport qu'en phase de contraction confirme la forte disponibilité de l'eau du sol qui, induisant des flux tardifs élevés et des flux nocturnes non nuis, conduisent simultanément la contrainte climatique, et à régéner totalement le diamètre des branches.

\section{Cas d'une ETP journalière faible}

$\mathrm{Si}$, dans les deux traitements, les cinétiques de mobilisation des deux sources d'eau au courant de transpiration apparaissent relativement synchrones, des différences importantes apparaissent quant aux valeurs des phénomènes observés (fig 7). Durant la première phase de décroissance des diamètres (7-12 heures) les rapports précédents définis sont respectivement égaux à -877 (NI) et à $-73 \mu \mathrm{m} \cdot \mathrm{mm}^{-1}$ (I). Ces valeurs montrent que les réserves en eau de l'arbre NI sont mobilisées de façon beaucoup plus intense que dans le cas précédent, bien que la demande climatique durant cette même période de temps soit plus faible : $296 \mathrm{w} \cdot \mathrm{m}^{-2}$ ce jour contre $478 \mathrm{w} \cdot \mathrm{m}^{-2}$ le jour précédemment décrit. Ce fait confirme que la décroissance continue de la disponibilité de l'eau dans le sol avec le temps est à l'origine d'une sollicitation accrue des réserves en eau de l'arbre dans l'évapotranspiration. Inversement, dans l'arbre I, la participation des flux racinaires à la compensation de la contrainte climatique est relativement plus importante, les rapports instantanés « demande climatique - disponibilité de l'eau du sol » étant plus faibles. Dans la phase intermédiaire de faible ETP (12-16 heures) les rapports précédents sont respectivement égaux à 520 et $72 \mu \mathrm{m} \cdot \mathrm{mm}^{-1}$. Ils traduisent que l'extraction racinaire, qui dans les deux cas doit encore satisfaire à la demande climatique, a des effets différents, selon les traitements, sur la régénération des réserves en eau des arbres précédemment utilisées : les taux de reconstitution sont respectivement de 29 et $70 \%$ dans les arbres NI et I. Avec l'apparition d'une nouvelle demande climatique forte (17-18 heures), la participation des réserves de l'arbre NI apparaît plus faible qu'en début de journée (rapport égal à $155 \mu \mathrm{m} \cdot \mathrm{mm}^{-1}$ ), leur niveau dans l'arbre étant plus faible. Cette participation est de plus rapidement stabilisée. Pour l'arbre irrigué cette participation d'abord élevée diminue dès 17 heures, les flux racinaires devenant importants sous l'influence de l'irrigation. En phase terminale il existe une reconstitution totale des réserves en eau des arbres, plus tardive en l'absence d'irrigation. On note in fine un accroissement important des diamètres sous irrigation. L'évolution dans le temps de l'indice précédemment défini, évolution du diamètre des branches/flux de sève, apparaît comme un indice pouvant caractériser l'évolution de la participation relative des deux sources d'eau, réserves en eau de l'arbre et eau du sol, à la satisfaction de la contrainte climatique.

\section{CONCLUSIONS}

L'utilisation simultanée de la mesure des flux thermiques et de la microdendrométrie, couplées à l'étude des transferts de l'eau dans le sol, permet de différencier à la fois le fonctionnement hydrique interne de l'arbre et son comportement hydrique global en fonction de son alimentation en eau et des modalités d'apport de celle-ci au sol : la fluximétrie thermique donne accès à la quantification des flux élémentaires de sève brute traversant les différentes plages de la section du tronc, au flux global traversant celui-ci et aux cinétiques nycthémérales les caractérisant ; la microdendométrie rend compte d'une part des conditions de mobilisation des réserves en eau propres des parties ligneuses de l'arbre au courant de transpiration, de l'intensité de cette mobilisation et des conditions de leur reconstitution sous l'effet des flux racinaires tardifs et nocturnes. Leur utilisation simultanée permet ainsi de détecter : i) un déficit d'alimentation hydrique de l'arbre et l'hétérogénéité des flux de sève entre arbres ; ii) l'existence d'une sectorisation importante des flux de sève 
brute dans la section des trones indiquant l'existence de connexions exclusives racines-secteur du tronc, et de connexions peu ou pas marquées avec les branches en relation avec les secteurs du tronc ; iii) la participation relative des deux sources d'eau, eau du sol et réserves en eau de l'arbre à l'évapotranspiration ; iv) le devenir de ces réserves.

Il apparaît donc que l'utilisation en continu, puisque automatisable, de la méthodologie proposée constitue une approche intéressante dans l'étude du comportement hydrique des arbres fruitiers. Cependant la mise en évidence d'une sectorisation des flux dans le tronc et de possibles perturbations du développement racinaire, ces dernières impliquant que, sous irrigation localisée, une racine supposée en zone humectée se trouve en zone sèche ou inversement, nécessite que les mesures de flux de sève locales soient faites à différentes positions autour du tronc pour estimer le flux global traversant les troncs. Cette obligation peut devenir, en multipliant le nombre de mesures à faire, une limite importante à l'utilisation des mesures de flux de sève locales.

Cette méthodologie offre néanmoins des éléments essentiels d'analyse permettant, dans les vergers en irrigation localisée, notamment si celle-ci est rationnée, de juger des besoins en eau des arbres et de l'efficacité de l'irrigation pratiquée. Elle devrait permettre d'améliorer les conditions de mise en ouvre de l'apport d'eau concernant notamment le choix de l'heure de déclenchement de l'apport d'eau au verger par rapport à l'évolution de la demande climatique horaire, la fixation de la dose d'irrigation minimale en irrigation rationnée, le rythme du fractionnement des apports d'eau, la fixation du nombre nécessaire de goutteurs et de leur positionnement par rapport aux arbres Elle devrait également permettre d'affiner l'étude de l'évolution du rapport variation du diamètre/flux de sève et de sa gamme de variation pour un verger donné, dans la période journalière de croissance de la demande climatique, et de vérifier si ce rapport peut constituer un critère susceptible de contrôler l'irrigation et son efficacité.

La facilité de mise en ouvre de ces deux techniques expérimentales devrait aussi permettre d'obtenir, sans une forte augmentation des moyens expérimentaux à mettre en cuvre, des séries importantes de résultats de mesures permettant d'analyser des modalités très variées.

\section{RÉFÉRENCES}

Cabibel B (1994) Continuité des flux hydriques dans le système sol-racines-tronc. Cas des arbres fruitiers. agronomie 14, 503-514

Cabibel B, Do F (1991) Mesures thermiques des flux de sève dans les troncs et les racines et fonctionnement hydrique des arbres. I. Analyse théorique des erreurs sur la mesure des flux et validation des mesures en présence de gradients thermiques extérieurs. agronomie 11, 669-678

Do F (1987) Caractérisation spatiale de l'extraction racinaire par la mesure des flux de sève brute en conditions initiales d'humectation homogène du sol. DEA de science agronomique, Ensa Montpellier USTL Montpellier II - Inra Avignon

Granier A (1985) Une nouvelle méthode pour la mesure du flux de sève brute dans le tronc des arbres. Ann Sci For 2, 193-200

Huguet JG (1985) Appréciation de l'état hydrique d'une plante à partir des variations micrométriques de la dimension des fruits et des tiges au cours de la journée. agronomie 5, 733-741

Isbérie C (1992) Contribution du sol à l'alimentation hydrique d'un verger de cerisiers micro-irrigué selon un pilotage tensiométrique. Thèse, univ Montpellier-II, Cemagref, 415

Kesner CD, Hahn BR, Klein WM, Bralts VF (1985) Nitrogen application with trickle irrigation on sour cherry trees. Drip/Trickle Irrig. Action, Proc. Third Int. Drip/Trickle Irrig Cong, 18-21 Nov 1985 , Fresno, CA, États-Unis, ASAE Saint Joseph, MI, États-Unis, vol.1, 350-356

Normand M (1974) Méthode d'étalonnage d'un humidimètre à neutrons utilisant les mesures de densité du densimètre gamma associé. IAEA-SM-176/24, 53-69

Peyremorte P (1980) Des tensiomètres pour améliorer la conduite de l'irrigation localisée. Eau aménagement région provençale 26

Sizov SS, Iastreb GV (1976) Régime hydrique du cerisier dans les conditions d'irrigation du sud de l'Ukraine. Nauch. Dokl. Vyssch. Shk. Biol. Nauky, SSSR, 142-143

Smith MW, Kenworthy AL (1979) The response of fruit trees in Michigan to trickle irrigation. Commun Soil Sci Plant Anal 10, 1371-1380

Soing P, Mandrin JF (1992) Aspersion, goutte à goutte et rationnement sur cerisier. Infos-Ctifl 79, 33-36 